\title{
The Effects of Antiretroviral Drugs on the Absorbance Characteristics of HIV-Infected Blood
}

\author{
Okwuchukwu Ani', Sam Omenyi², Chinonso Achebe ${ }^{2}$ \\ ${ }^{1}$ Department of Mechanical and Production Engineering, Enugu State University of Science and Technology, \\ Agbani, Nigeria \\ ${ }^{2}$ Department of Mechanical Engineering, Nnamdi Azikiwe University, Awka, Nigeria \\ Email: chinobert2k@yahoo.com
}

Received 3 July 2015; accepted 1 September 2015; published 4 September 2015

Copyright @ 2015 by authors and Scientific Research Publishing Inc.

This work is licensed under the Creative Commons Attribution International License (CC BY).

http://creativecommons.org/licenses/by/4.0/

(c) (i) 0 pen Access

\section{Abstract}

In the twenty first century research works, there may be a need to achieve a more reliable research result through a synergy between engineers and biological researchers. The peak absorbance data for various interacting systems were measured. These were used to show that the antiretroviral drug has the effect of increasing the peak absorbance values of both the uninfected and infected blood components, i.e., the drugs are made able to increase the light absorption capacity of the blood cells. For drug 2 that contains three components including Efavirenz, the drug effect on lymphocytes was increased by about $38 \%$ for patients that had been on antiretroviral drug treatment. Mathematical models were proposed and used in determining the coating effectiveness of antiretroviral drugs in the presence and absence of HIV. The use of the findings of this work by pharmaceutical industries may help in the search for more effective antiretroviral drugs for the treatment of HIV patients.

\section{Keywords}

Absorbance, Transmittance, Dielectric Constant, Human Immunodeficiency Virus, Antiretroviral Drug, Coating Effectiveness, Lymphocyte, Wavelength

\section{Introduction}

The HIV/AIDS cases have hitherto been managed clinically with the discovery and administration of Highly Active Anti-retroviral Therapy (HAART). But these anti-retroviral drugs are heavily attacked and resisted by the 
HIV in the human system because they are DNA-based while the HIV is RNA-based. Hence, the ineffectiveness and failure of HAART are as a result of the ability and capacity of HIV to develop resistance to the administered anti-retroviral drugs even when the combination therapy is for the HIV patient. The discovery and application of highly active anti-retroviral therapy (HAART) to suppress HIV have revolutionized the clinical management of HIV/AIDS cases. The HIV however, has the capacity to develop resistance to the antiretroviral drugs and this phenomenon has turned out to be a significant cause of failure of HAART [1]. HIV, which is a rapidly mutating RNA-based virus, lacks the ability to checkmate the possible genetic mutations that can occur during replication. Hence, this rapid genetic variation has become the major factor for which this menace has consistently defiled clinical solutions.

The increasing rate of HIV infection globally is blamed on the ineffectiveness of some available antiretroviral therapy to block or resist perfectly this virus from invading the uninfected white blood cells. The mechanism by which drugs can block the virus seems weak and hence it becomes necessary to study the interaction between the HIV and the drug-coated white blood cells. The problem of formulating drugs that can summarily eliminate HIV, remains a challenge. The question arises as to how effective the available antiretroviral drugs are. The answer to such question may be found by studying surface effects in HIV-drug interactions.

There are several classes of drugs, which are usually used in combination, to treat HIV infection. Use of these drugs in combination can be termed anti-retroviral therapy (ART), combination anti-retroviral therapy (cART) or highly active anti-retroviral therapy (HAART). Anti-retroviral (ARV) drugs are broadly classified by the phase of the retrovirus life-cycle that the drug inhibits. Typical combinations include 2 NRTIs as a "backbone" along with 1 NNRTI or 1 PI as a "base" [2].

Since some of the drugs act as blockers, the blocking would be effective if the drug completely coats the cells. The extent of the cell surface that is coated is important.

We intend to use the concept of absorbance to address this issue. Achebe and Omenyi [3] have shown that absorbance is a surface phenomenon. They showed that the peak absorbance of the surface of each blood component was reduced by the presence of the virus. The question now arises as to what extent the peak absorbance of the surface of a given HIV infected blood component changed by the administration of the anti retroviral drugs.

\section{Methodology}

\subsection{Sample Collection}

The popular and commonly used unexpired Antiretroviral drugs (three single tablets and two HAART), from the University of Nigeria Teaching Hospital (UNTH) APIN CENTRE PEPFAR, Ituku-Ozalla, Enugu State were collected (see Figure 1). Table 1 shows the details of the five different antiretroviral drugs used in the study.

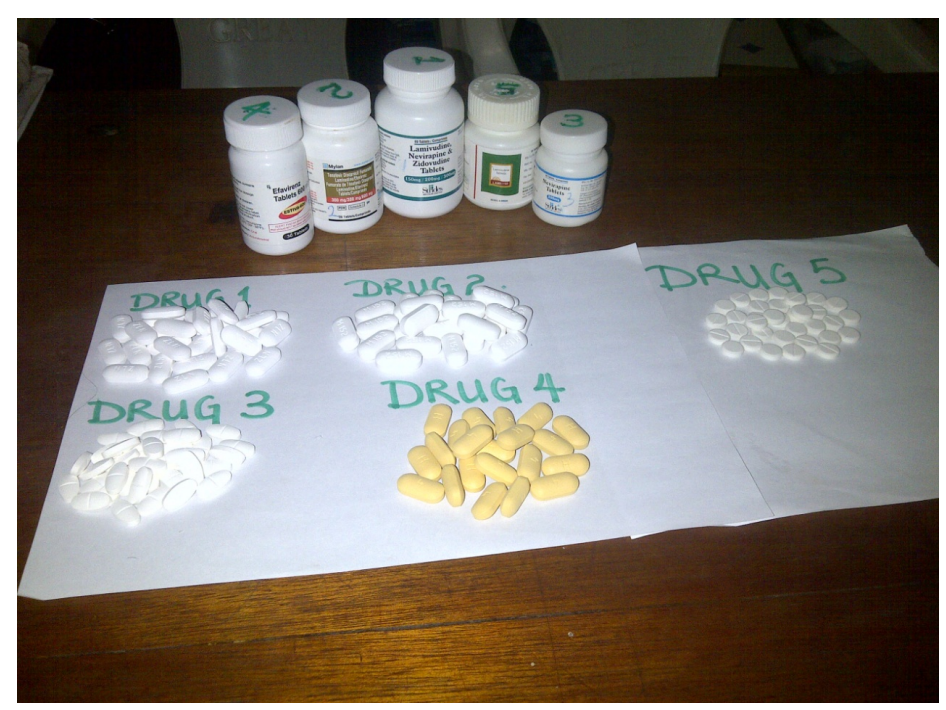

Figure 1. The tablets of the five different antiretroviral drugs used. 
Table 1. The details of the five different antiretroviral drugs used in the study.

\begin{tabular}{|c|c|c|c|c|c|c|}
\hline $\begin{array}{c}\text { Drug } \\
\text { Number }\end{array}$ & Tablets & Abbreviation & Size & $\begin{array}{l}\text { Batch } \\
\text { Number }\end{array}$ & $\begin{array}{l}\text { Expiration } \\
\text { Date }\end{array}$ & $\begin{array}{l}\text { Pharmaceutical } \\
\text { Company }\end{array}$ \\
\hline 1 & $\begin{array}{l}\text { Lamivudine, } \\
\text { Nevirapine \& } \\
\text { Zidovudine }\end{array}$ & $3 \mathrm{TC}+\mathrm{NVP}+\mathrm{ZDV}$ & $\begin{array}{c}150 \\
\mathrm{mg} / 200 \mathrm{mg} / 300 \mathrm{mg}\end{array}$ & 7220929 & 01/2016 & $\begin{array}{l}\text { Strides Arcolab } \\
\text { Limited }\end{array}$ \\
\hline 2 & $\begin{array}{l}\text { Tenofovir, } \\
\text { Lamivudine \& } \\
\text { Efavirenz }\end{array}$ & $\mathrm{TDF}+3 \mathrm{TC}+\mathrm{EFV}$ & $\begin{array}{c}300 \\
\mathrm{mg} / 300 \mathrm{mg} / 600 \mathrm{mg}\end{array}$ & 3018522 & 09/ 2015 & $\begin{array}{c}\text { Mylan Laboratories } \\
\text { Limited }\end{array}$ \\
\hline 3 & Nevirapine & NVP & $200 \mathrm{mg}$ & 7216348 & $04 / 2015$ & $\begin{array}{l}\text { Strides Arcolab } \\
\text { Limited }\end{array}$ \\
\hline 4 & Efavirenz & $\mathrm{EFV}$ & $600 \mathrm{mg}$ & E121035A & 07/2015 & $\begin{array}{l}\text { HETERO LABS } \\
\text { LIMITED }\end{array}$ \\
\hline 5 & Lamivudine & 3TC & $150 \mathrm{mg}$ & LEX - 023 & 04/ 2016 & $\begin{array}{l}\text { MCNEIL \& } \\
\text { DRUGS } \\
\text { Pharmaceuticals } \\
\text { Ltd. }\end{array}$ \\
\hline
\end{tabular}

Drugs 1 and 2 are both Highly Active Antiretroviral Therapy (HAART) as well as Fixed Dose Combination (FDC), while drugs 3, 4 and 5 are single antiretroviral drugs. Drugs 1, 3 and 5 are administered to HIV patients twice daily while drugs 2 and 4 are taken once a day. It is worthy to note that all the antiretroviral drugs used were not expired during the period of the experiments.

Blood samples were collected from Nnamdi Azikiwe University Teaching Hospital (NAUTH) Nnewi and Anambra State Teaching Hospital, Amaku with an Ethical Clearance certificate as follows:

- from ten HIV infected persons who were not yet on HIV drugs,

- from ten infected persons that had already commenced treatment with antiretroviral drugs and

- from ten HIV negative persons.

Altogether, a total of thirty samples from different individuals were collected using 5 ml syringe, and screened to determine the infection status and stored in anticoagulant test tubes and ice packs to ensure the freshness and to avoid the samples becoming lysed. The samples were thereafter stored in a refrigerator for proper preservation. Note that the Ethical Clearance Certificate obtained from the hospitals for the thirty blood samples collected from different individuals also depicts that an informed consent was obtained from those individuals before the test.

\subsection{Sample Preparation}

The drugs passed through serial dilution at Tahilah Diagnostic Laboratories, Awka, in order to get the right concentration of drug in the blood. After the serial dilutions to $10^{-2}$, the drug solution mixed with the blood was incubated at normal body temperature $\left(37^{\circ} \mathrm{C}\right)$ to facilitate drug-blood interactions (This is an in vitro experiment) [4]. The knowledge of the onset and duration of action of each drug was used in administering the start dose and the maintenance dose in the blood samples. These collected samples with drug concentrations were loaded into a centrifugal separator and the blood components were separated at Tahilah Diagnostic Laboratories, Awka. The blood samples were spun at the speed of $3000 \mathrm{rpm}$ for 5 minutes in the centrifuge. It works by the principle of centrifugal force which is the outward pull due to rotation exerted by the centrifuge which is greater than the force of gravity thereby causing the particles in the fluid to sediment. This helped to obtain such components as White Blood Cells (WBC) also called the Lymphocytes, Red Blood Cells (RBC), and the Plasma or Serum, each sample at a time. Glass slides were prepared and smeared with the samples for absorbance measurements. The slide preparations and sample smearing were done at the same laboratory. About 600 slides were successfully prepared in the laboratory.

\subsection{Measurements}

Absorbance measurements were done on all the different components of thirty samples (HIV infected blood of ones that had not started ARV treatment, HIV infected blood of those that had started ARV treatment and unin- 
fected blood samples). A digital Ultraviolet Visible MetaSpecAE1405031Pro Spectrophotometer was used at the laboratory of the Department of Mechanical Engineering, Nnamdi Azikiwe University, Awka for the measurements. The absorbance values of the samples were measured over a range of wavelength between 230 and 800 Hertz alongside with their corresponding transmittance values.

\section{Results and Analysis}

\subsection{Infected Persons That Had Already Commenced Treatment with Antiretroviral Drugs}

The absorbance values measured for each of the ten samples were averaged; it was the average values for each blood component incubated in each antiretroviral drug for those that had previously started drug treatment that were plotted as a function of the wavelength, as given in Figure 2.

Figure 2 gives the absorbance results for drugs 1 to 5 in the HIV positive blood components with prior treatment with antiretroviral drug. The absorbance of the interacting systems significantly increased as the wavelengths increased until a peak absorbance was reached at $320 \AA$ for the Lymphocytes and Plasma, and $410 \AA$ for the Red blood cells and the Whole blood. Further increase in the wavelength gave rise to a decrease in the absorbance values which became almost constant between wavelengths 600 and $800 \AA$. The peak values fall within the visible range of the ultraviolet radiation. The peak absorbance values are presented in Table 2 together with the corresponding wavelengths.

The peak absorbance values for the five antiretroviral drugs on Lymphocytes range from 0.08 to 0.18 , on Plasma they range from 0.08 to 0.20 , on Red blood cells they range from 0.80 to 1.58 , and on Whole blood they range from 1.17 to 1.60 .

\subsection{HIV Infected Persons Who Were Not Yet on HIV Drugs}

The absorbance values measured for each of the ten samples were averaged; it was the average values for each blood component incubated in each antiretroviral drug for those that had not previously started drug treatment that were plotted as a function of the wavelength, are given in the preceding Figure 3.

Figure 3 gives the results for drugs 1 to 5 in the HIV positive blood components of patients that had not commenced antiretroviral treatment before this study. As observed before, the absorbance values significantly increased as the wavelengths increased until a peak absorbance was attained. Further increase in the wavelength gave sharp decrease in the absorbance values which remained almost constant between wavelengths 600 and $800 \AA$. The peak absorbance values for the five antiretroviral drugs on Lymphocytes range from 0.18 to 0.30 , on the Plasma they range from 0.08 to 0.28 , on Red blood cells they range from 0.60 to 2.10 , on Whole blood they range from 1.00 to 1.40 , falling within the visible range of the ultraviolet radiation which is 300 - $600 \AA$; these values are presented in Table 3 .

\subsection{HIV Negative Persons}

The absorbance values measured for each of the ten samples were averaged; it was the average values for each blood component incubated in each antiretroviral drug for HIV negative patients that were plotted as a function of the wavelength as given by [5]. The peak absorbance values from the work of Ani [5] are listed in Table 4.

Table 4 gives the data for absorbance for blood components of patients that were HIV negative. The peak absorbance values of the blood components with antiretroviral drugs are higher than the peak absorbance values of the blood components without antiretroviral drugs when Table 4 is compared with Table 2 and Table 3 . This indicates that the antiretroviral drug has the effect of increasing the peak absorbance values of the blood components, i.e., the drugs are made able to increase the light absorption capacity of the blood cells. Previous researches (Achebe, 2010) have shown that the virus reduces the peak absorbance values of the blood components.

This work compared the peak absorbance values of HIV positive blood and HIV negative blood and reported that the absorbance values of uninfected blood components were higher than those of infected blood components. The absorbance values of HIV positive samples are generally decreased by a significant factor. The apparent decrease in the absorbance of the HIV infected blood samples reveals the role of the virus in significantly affecting the surface properties of the infected blood cells. However, the restorative action of antiretroviral drugs is a positive sign to the reduction of the virus effect. This work is devoted to showing that the surface property 


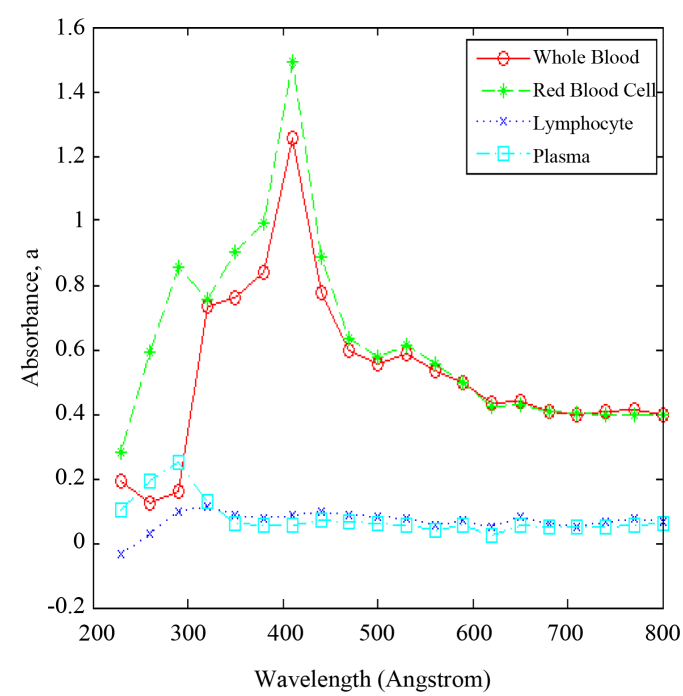

(a)

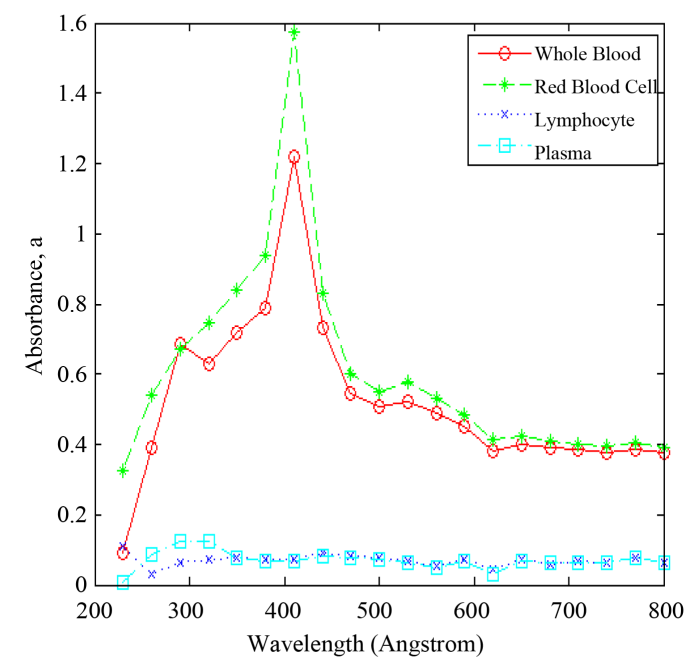

(c)

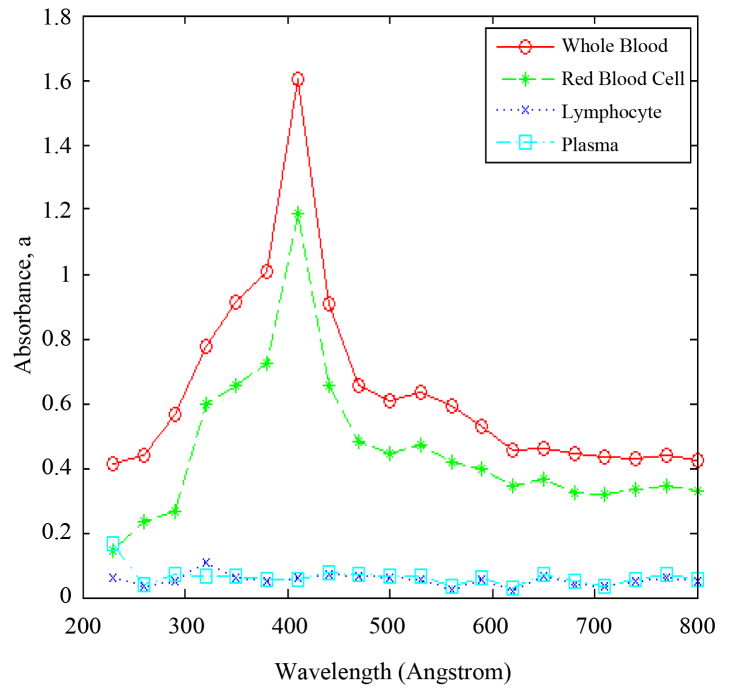

(b)

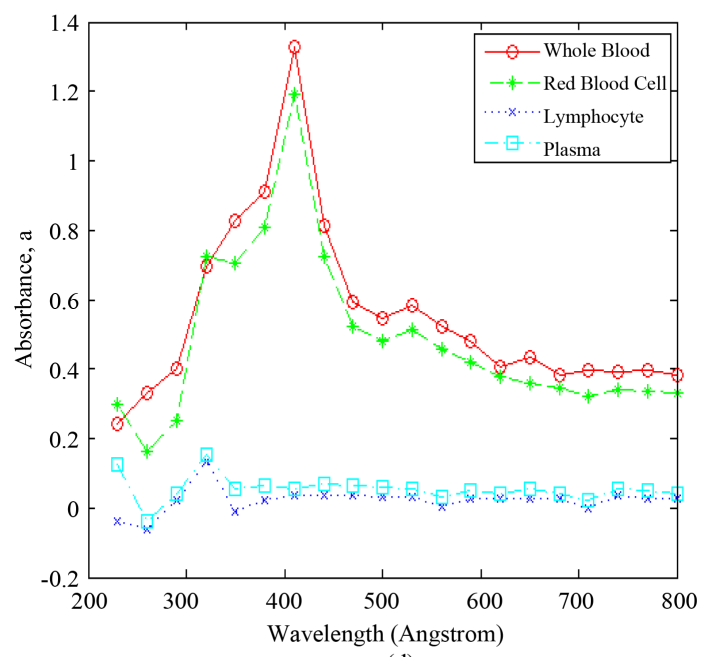

(d)

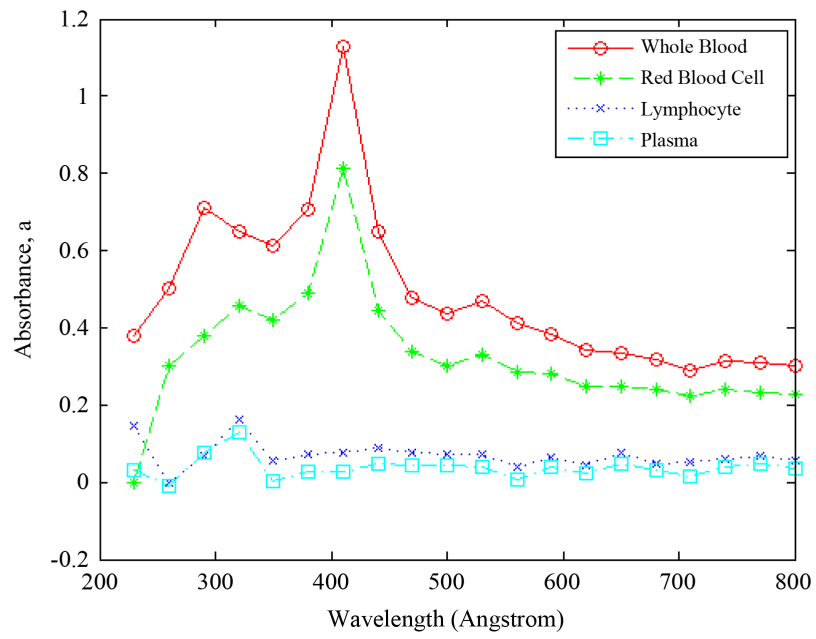

(e)

Figure 2. Absorbance, á versus Wavelength, $\lambda$ for HIV Positive Blood of patients that had commenced antiretroviral drug treatment before this study. (a) Drug 1; (b) Drug 2; (c) Drug 3; (d) Drug 4; (e) Drug 5. 


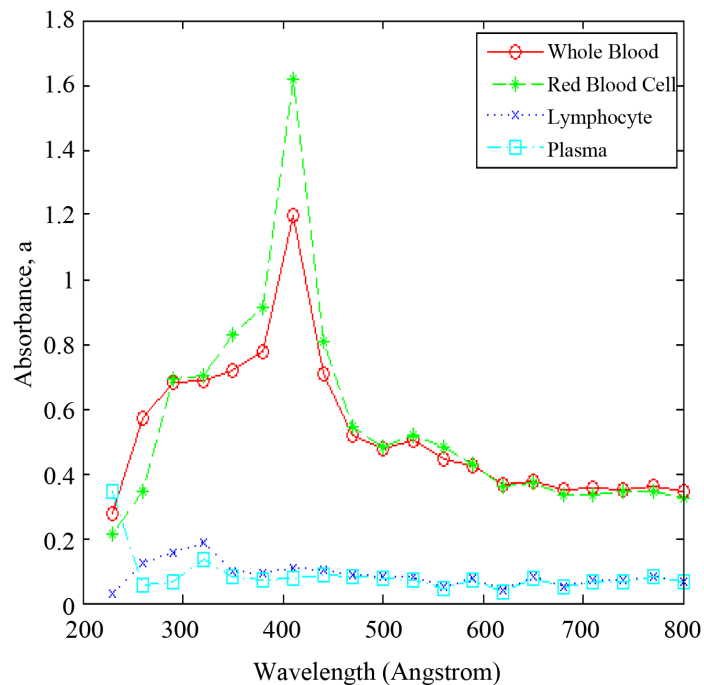

(a)

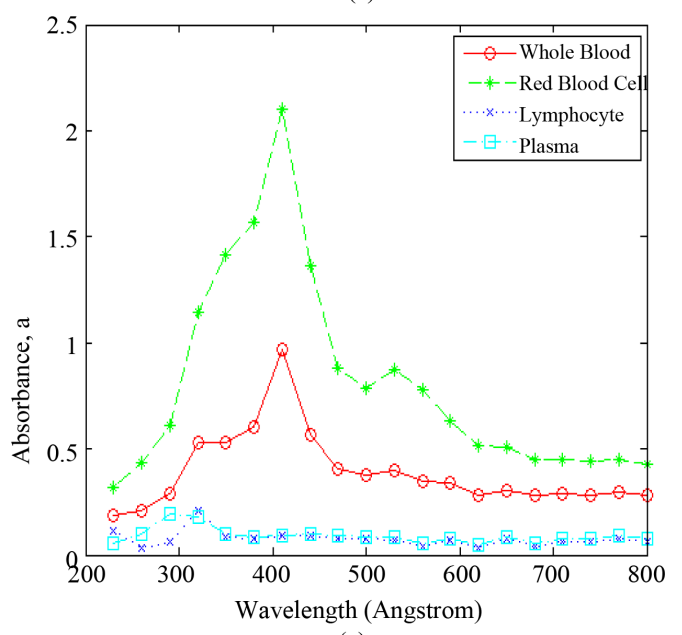

(c)

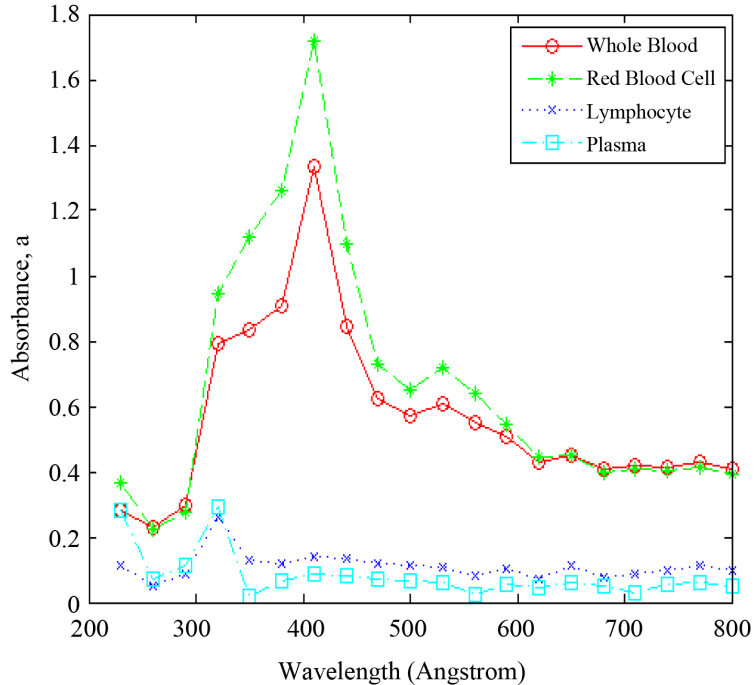

(b)

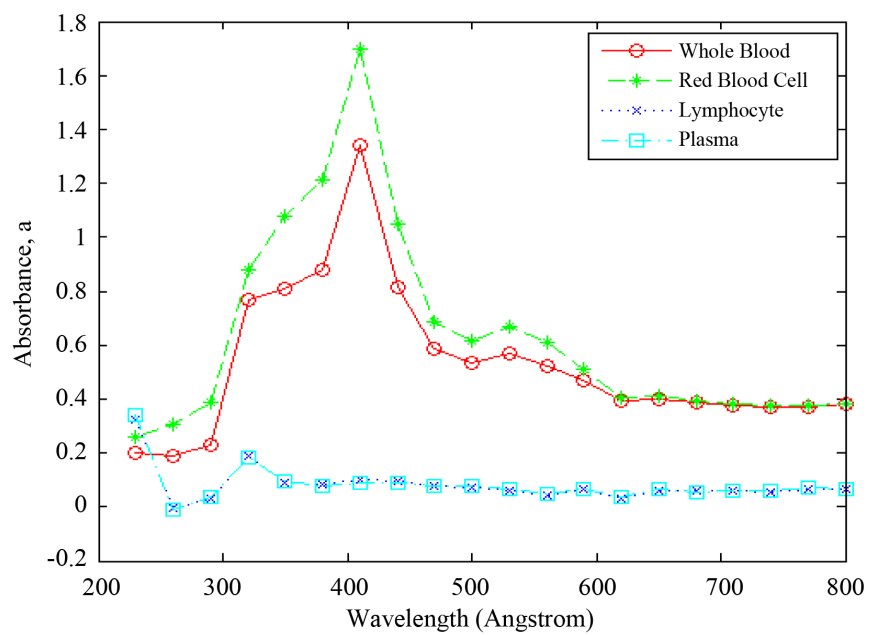

(d)

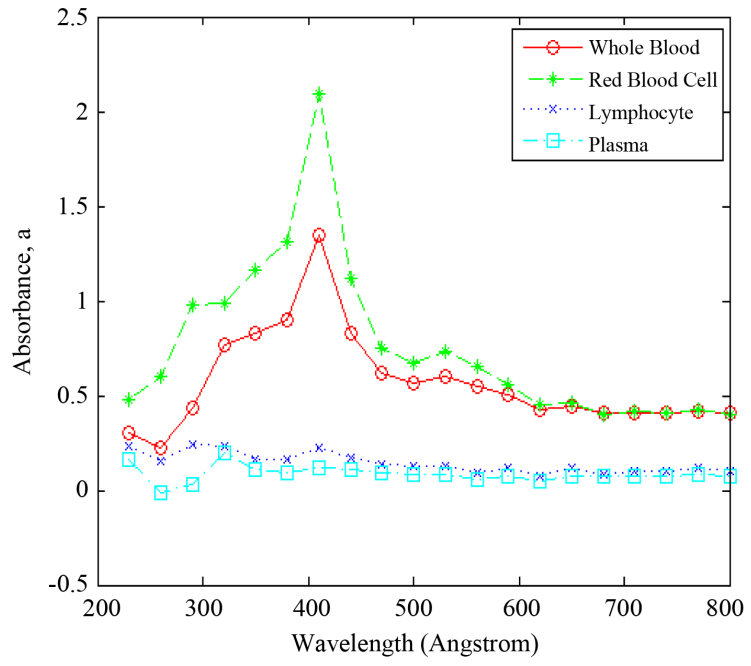

(e)

Figure 3. Absorbance, á versus Wavelength, $\lambda$ for HIV positive blood components of patients that had not previously commenced antiretroviral drug treatment. (a) Drug 1; (b) Drug 2; (c) Drug 3; (d) Drug 4; (e) Drug 5. 
Table 2. Data for absorbance for blood components of patients on drug treatment before this test.

\begin{tabular}{cccccccccc}
\hline \multirow{2}{*}{ Drug No. } & \multicolumn{2}{c}{ Whole blood } & \multicolumn{2}{c}{ RBC } & \multicolumn{2}{c}{ WBC } & \multicolumn{2}{c}{ Plasma } \\
\cline { 2 - 10 }$y$ (peak) & $\lambda, \AA$ & $\boldsymbol{a}$ (peak) & $\lambda, \AA$ & $\boldsymbol{a}$ (peak) & $\lambda, \AA$ & $\boldsymbol{a}$ (peak) \\
\hline 1 & 410 & 1.25 & 410 & 1.50 & 320 & 0.14 & 320 & 0.20 \\
2 & 410 & 1.60 & 410 & 1.20 & 320 & 0.10 & 320 & 0.08 \\
3 & 410 & 1.20 & 410 & 1.58 & 320 & 0.08 & 320 & 0.12 \\
4 & 410 & 1.36 & 410 & 1.20 & 320 & 0.17 & 320 & 0.18 \\
5 & 410 & 1.17 & 410 & 0.80 & 320 & 0.18 & 320 & 0.16 \\
\hline
\end{tabular}

Table 3. Absorbance data for blood components of patients not on drug treatment before this test.

\begin{tabular}{cccccccccc}
\hline \multirow{2}{*}{ Drug No. } & \multicolumn{2}{c}{ Whole blood } & \multicolumn{2}{c}{ RBC } & \multicolumn{3}{c}{ WBC } & \multicolumn{3}{c}{ Plasma } \\
\cline { 2 - 10 }$y$ (peak) & $\lambda, \AA$ & $\boldsymbol{a}$ (peak) & $\lambda, \AA$ & $\boldsymbol{a}$ (peak) & $\lambda, \AA$ & $\boldsymbol{a}$ (peak) \\
\hline 1 & 410 & 1.20 & 410 & 1.60 & 320 & 0.18 & 320 & 0.16 \\
2 & 410 & 1.36 & 410 & 1.76 & 320 & 0.26 & 320 & 0.08 \\
3 & 410 & 1.00 & 410 & 2.10 & 320 & 0.20 & 320 & 0.19 \\
4 & 410 & 1.40 & 410 & 2.08 & 320 & 0.30 & 320 & 0.28 \\
5 & 410 & 1.36 & 410 & 1.70 & 320 & 0.19 & 320 & 0.20 \\
\hline
\end{tabular}

Table 4. Data for absorbance for blood components of patients that were HIV negative.

\begin{tabular}{cccccccccc}
\hline \multirow{2}{*}{ Drug No. } & \multicolumn{2}{c}{ Whole blood } & \multicolumn{2}{c}{ RBC } & \multicolumn{2}{c}{ WBC } & \multicolumn{2}{c}{ Plasma } \\
\cline { 2 - 9 } & $\lambda, \AA$ & $\boldsymbol{a}$ (peak) & $\lambda, \AA$ & $\boldsymbol{a}$ (peak) & $\lambda, \AA$ & $\boldsymbol{a}$ (peak) & $\lambda, \AA$ & $\boldsymbol{a}$ (peak) \\
\hline 1 & 410 & 1.05 & 410 & 1.50 & 320 & 0.48 & 320 & 0.22 \\
2 & 410 & 1.40 & 410 & 1.88 & 320 & 0.52 & 320 & 0.18 \\
3 & 410 & 1.20 & 410 & 1.90 & 320 & 0.30 & 320 & 0.26 \\
4 & 410 & 1.24 & 410 & 2.28 & 320 & 0.28 & 320 & 0.30 \\
5 & 410 & 1.38 & 410 & 2.18 & 320 & 0.27 & 320 & 0.20 \\
\hline
\end{tabular}

of a blood component can be significantly changed by the antiretroviral drugs and that this effect can possibly be used in drug design.

\subsection{Test of Significance}

We study further the variation of peak absorbance between the three scenarios under consideration, for WBC which is the target for HIV, as extracted from Tables 2-4 and listed in Table 5.

The analysis of variance (ANOVA) for the three data sets gives the computed F ratio as 11.19 which is higher than the critical F ratio 3.88 for 0.05 level of significance. This shows that there are significant differences between the scenarios, A, B and C. To identify the scenario that is most significant analyses of the means of the data of Table 5 become necessary. Between scenarios A and B, the computed F ratio is 3.35 which is less than the critical $\mathrm{F}$ ratio of 4.75 . This shows that there is no significant difference between scenarios $\mathrm{A}$ and $\mathrm{B}$. Analyses of B and C (computed and critical F ratios are 8.20 and 4.75 respectively), and of A and C (computed and critical $\mathrm{F}$ ratios are 22.03 and 4.75, respectively) show there are significant differences between these pairs as presented in Table 5(a) and Table 5(b). These results show that scenario C, the absorbance of HIV negative white blood cells, is most significant. Thus, the light absorption capacities of the surfaces of the HIV negative white blood cells are significantly different from the light absorption capacities of HIV positive WBC whether or not they had earlier been subjected to antiretroviral drug treatment or not. This suggests that the antiretroviral drug has the effect of increasing the peak absorbance values of blood components, that is, the drugs are made to 
Table 5. Peak absorbance data for WBC for the three scenarios (ABC).

\begin{tabular}{ccccccc}
\hline & The scenarios & Drug 1 & Drug 2 & Drug 3 & Drug 4 & Drug 5 \\
\hline A & HIV + ve with drug pretreatment & 0.14 & 0.10 & 0.08 & 0.17 & 0.18 \\
B & HIV + ve without drug pretreatment & 0.18 & 0.26 & 0.20 & 0.30 & 0.19 \\
C & HIV - ve & 0.48 & 0.52 & 0.30 & 0.28 & 0.27 \\
\hline
\end{tabular}

increase the light absorption capacity of the blood cells while HIV has the effect of reducing the peak absorbance values of blood components, that is, the virus has the ability to reduce the light absorption capacity of the blood cells.

\section{Discussions}

Three questions can arise in the analysis of the data above. What is the effect of antiretroviral drug treatment on the absorbance of blood component? What is the effect of the HIV on the absorbance of a blood component? And what is the combined effect of the drug and HIV on the surface of the blood component? These questions have somehow been answered above but additional work is presented through mathematical models proposed to estimate these effects.

\subsection{Antiretroviral Drug Effect, $\eta_{d}$}

The effect of the antiretroviral drug on the surface of a blood cell can be estimated from.

$$
\eta_{d}=\frac{\tilde{a}_{d}-\tilde{a}_{o}}{\tilde{a}_{d}}
$$

where $\tilde{a}_{d}$ is peak absorbance for drug film on blood component, and

$\tilde{a}_{o}$ is peak absorbance for blood component alone

Equation (1) is actually saying that, from absorbance concept, the difference the drug film makes in the absorbance of a blood component when compared with that of the absorbance of the blood component alone is some measure of drug effect. The antiretroviral drug has the capacity to increase the absorbance of a given blood component surface. The values of $\eta_{d}$ are computed and reported on Table 5.

\subsection{HIV Effect, $\eta_{h}$}

The effect of HIV on the surface of a blood component in the presence of the antiretroviral drug, can be estimated from

$$
\eta_{h}=\frac{\tilde{a}_{d}-\tilde{a}_{h}}{\tilde{a}_{d}}
$$

HIV has the effect of reducing the absorbance of the surface of a given blood component. The amount by which the surface of a blood component surface is decreased by HIV will be given by Equation (2). If HIV fails to reduce the absorbance of a drug film coated blood surface, it means that the HIV has not attached itself to the surface, then $\tilde{a}_{h}=\tilde{a}_{d}$ and the effect of HIV will be zero. Values of $\eta_{h}$ are presented in Table 6.

\subsection{Combined Effect of Antiretroviral Drug and HIV, $\eta_{d h}$}

To determine the combined effect of both HIV and administered antiretroviral drug, consider Equations (1) and (2), and obtain Equation (3). This will give us the idea of the effect of both the HIV and the antiretroviral drugs acting together on the blood and its components. The calculated values are listed in Table 7.

$$
\eta_{d h}=\eta_{d} \eta_{h}
$$

\subsection{Results}

Using the relevant data together with the proposed expressions, the effects were calculated as presented in 
Table 6. Effects of drug film coating n blood component, $\eta_{h}$.

\begin{tabular}{cccc}
\hline \multirow{2}{*}{ Drug } & \multicolumn{3}{c}{ Uninfected Blood Components } \\
\cline { 2 - 4 } & WBC & RBC & Plasma \\
\hline 1 & 0.8163 & -0.1626 & 0.6632 \\
2 & 0.8304 & 0.0724 & 0.5883 \\
3 & 0.7060 & 0.0822 & 0.7150 \\
4 & 0.6850 & 0.2351 & 0.7530 \\
5 & 0.6733 & 0.2000 & 0.6295 \\
\hline
\end{tabular}

Table 7. Effect of HIV on drug coated blood component, $\eta_{h}$.

\begin{tabular}{|c|c|c|c|c|c|c|c|c|c|}
\hline \multirow{2}{*}{ Drug } & \multicolumn{3}{|c|}{$\begin{array}{l}\text { HIV infected without previous drug } \\
\text { treatment }\end{array}$} & \multicolumn{3}{|c|}{ HIV infected with previous drug treatment } & \multicolumn{3}{|c|}{ Uninfected Blood Components } \\
\hline & WBC & RBC & Plasma & WBC & $\mathrm{RBC}$ & Plasma & WBC & $\mathrm{RBC}$ & Plasma \\
\hline 1 & 0.6250 & -0.0667 & 0.2727 & 0.7083 & 0.0000 & 0.0909 & 0.8163 & -0.1626 & 0.6632 \\
\hline 2 & 0.5000 & 0.0638 & 0.5556 & 0.8077 & 0.3617 & 0.5556 & 0.8304 & 0.0724 & 0.5883 \\
\hline 3 & 0.3333 & -0.1053 & 0.3333 & 0.7333 & 0.1684 & 0.5385 & 0.7060 & 0.0822 & 0.7150 \\
\hline 4 & 0.0357 & 0.0877 & 0.0357 & 0.3929 & 0.4737 & 0.4000 & 0.6850 & 0.2351 & 0.7530 \\
\hline 5 & 0.2963 & 0.2202 & 0.2963 & 0.3333 & 0.6330 & 0.4074 & 0.6733 & 0.2000 & 0.6295 \\
\hline
\end{tabular}

Tables 6-8.

Table 6 shows the effects of coating on uninfected blood components with the antiretroviral drugs. The effects on Red blood cells gave inconsistent results, one negative and the rest are positive values which are comparatively small. These suggest that the drugs do not have any effect on the red blood cell surfaces. Note that the drugs were specifically designed to affect the surfaces of white blood cells which are normally targeted by HIV. So, no relevant and reliable effect was actually expected [6]. The coating effects for the white blood cells and plasma are positive for five different antiretroviral drugs. Note also that the drugs are in solution in the plasma and so are bound to affect its property.

Drug 2 gave the highest effect for the White blood cells, followed by drug 1, while drug 5 gave the lowest value. Efavirenz is usually administered once daily; it may most likely deposit a thick layer of its film on the Lymphocytes. We can infer that the regimen of drug 2 that contains Efavirenz in combination would possibly be the most effective when compared with the other antiretroviral drugs if thick drug film contributes to effective blocking of HIV. The preference of HAART or regimen with Efavirenz combination is because of its fast viral suppression, easy compliance because of the dosage (i.e. taken once a day), it is best for HIV patients with co-infections like HIV-TB, HIV-Malaria, HIV-Hepatitis, and the pregnant HIV patients are now treated with it because it is not harmful to the foetus. The effect values for drugs 1 and 2 are higher than those of drugs 3, 4 and 5. Drugs 1 and 2 are HAART while drugs 3, 4 and 5 are single drugs. Some research findings of the biological researchers show that HAART (a regimen that contains three antiretroviral drugs from two different classes of antiretroviral drugs) are clinically more effective. This is in agreement with results of Table 6 that show them to have the highest effect.

Table 7 shows the effects of coating of the infected blood components with the antiretroviral drugs. The effectiveness of coating of the antiretroviral drugs on Red blood cells gave varied results, two negative and the rest are very low positive values. These suggest, as stated before, that the drugs do not have any effect on the red blood cells surfaces. Note that the drugs were specifically designed to block HIV from attacking the white blood cells. So, no relevant and reliable effect was actually expected [6]. The values of coating effectiveness for the white blood cells and plasma are positive for five different antiretroviral drugs. Note also that the drugs are in solution in the plasma and so are bound to affect its property, as stated before.

The values for drugs 1 and 2 are higher than that of drugs 3, 4 and 5 as also noted in Table 5. Drugs 1 and 2 
are HAART while drugs 3, 4 and 5 are single drugs. Some research findings of the biological researchers show that HAART (a regimen that contains three antiretroviral drugs from two different classes of antiretroviral drugs) are clinically more effective. Note also that the effect is more pronounced for the cases of patients that were in antiretroviral drug treatment before the samples were taken (81\%) when compared with those that had not commenced any treatment (50\%), for drug 2 cases. In this case, increase in absorbance was about 38\%, and varies with each drug. (We wish to inform that the type of drug administered to patients before this study, was not known).

Table 8 shows the combined effect of coating of the infected blood components with the antiretroviral drugs. The effectiveness of coating of the antiretroviral drugs on Red blood cells gave varied results, as previously stated. Here, they are so small that it is clear that the antiretroviral drugs do not have any discernible effect on the red blood cells. The values of coating effectiveness for the white blood cells and plasma are all positive for five different antiretroviral drugs with those for white blood cells being generally higher.

The values for drugs 1 and 2 are consistently higher than those of drugs 3, 4 and 5, confirming earlier conclusions. For drug 2, the combined effect was about $67 \%$ for blood of patients who were undergoing treatment and $42 \%$ for those that had not commenced treatment. The pharmaceutical companies that produced these drugs have tested them and were specific on what each drug can do. The report presented here is not aimed at informing these companies about their drugs, but our main aim is to show that from a different approach, it is possible to study the effectiveness of the drugs.

\subsection{Test of Significance}

It is important study the data on combined coating effectiveness for the three scenarios for the lymphocytes which are targets for HIV. The relevant data are presented in Table 9.

The analysis of variance (ANOVA) for the three sets of data in Table 9 gives the computed F ratio as 10.33 which is higher than the critical $F$ ratio of 3.88 for 0.05 level of confidence. Thus, there are significant differences between the means of the data for scenarios, A, B and C. To identify the scenario that is most significant, analyses of the means of these data become necessary. Between scenarios A and B, the computed F ratio is 2.87 which is less than the critical $\mathrm{F}$ ratio of 4.75 . This shows that there is no significant difference between scenarios A and B. Analyses of A and C (computed and critical F ratios are 7.87 and 4.75 respectively), and of B and C (computed and critical $\mathrm{F}$ ratios are 20.2 and 4.75, respectively) show that there are significant differences between the means of these pairs of data. These results show therefore that scenario $\mathrm{C}$, the combined drug coating effectiveness of HIV negative white blood cells, is most significant. Thus, the coverage of the surfaces of the HIV negative white blood cells by drug films is more effective than the coverage of HIV positive WBC cell

Table 8. Combined effect of coating, $\eta_{d h}$.

\begin{tabular}{ccccccccccc}
\hline & \multicolumn{3}{c}{$\begin{array}{c}\text { HIV infected without } \\
\text { previous drug treatment }\end{array}$} & \multicolumn{3}{c}{$\begin{array}{c}\text { HIV infected with previous } \\
\text { drug treatment }\end{array}$} & \multicolumn{3}{c}{ Uninfected Blood Components } \\
\cline { 2 - 11 } & WBC & RBC & Plasma & WBC & RBC & Plasma & WBC & RBC & Plasma \\
\hline 1 & 0.5102 & 0.0108 & 0.1809 & 0.5782 & 0.0000 & 0.0535 & 0.8163 & -0.1626 & 0.6632 \\
2 & 0.4152 & 0.0005 & 0.3269 & 0.6707 & 0.0262 & 0.3269 & 0.8304 & 0.0724 & 0.5883 \\
3 & 0.2353 & -0.0009 & 0.1925 & 0.5177 & 0.0138 & 0.3850 & 0.7060 & 0.0822 & 0.7150 \\
4 & 0.0245 & 0.0206 & 0.0502 & 0.2691 & 0.1114 & 0.3012 & 0.6850 & 0.2351 & 0.7530 \\
5 & 0.1995 & 0.0440 & 0.1632 & 0.2244 & 0.1266 & 0.2565 & 0.6733 & 0.2000 & 0.6295 \\
\hline
\end{tabular}

Table 9. Analysis of combined drug coating effectiveness for lymphocytes at the different scenarios.

\begin{tabular}{ccccccc}
\hline & The scenarios & Drug 1 & Drug 2 & Drug 3 & Drug 4 & Drug 5 \\
\hline A & HIV + ve with drug pretreatment & 0.5782 & 0.6707 & 0.5177 & 0.2691 & 0.2244 \\
B & HIV + ve without drug pretreatment & 0.5102 & 0.4152 & 0.2353 & 0.0245 & 0.1995 \\
C & HIV - ve & 0.8163 & 0.8304 & 0.7060 & 0.6850 & 0.6733 \\
\hline
\end{tabular}


surfaces whether or not they had earlier been subjected to antiretroviral drug treatment or not. The drug films adhere more effectively on surfaces of blood cells not infected by HIV. This suggests that HIV has the effect of reducing the surface area of blood cells, which has previously been reported [1] [3] [5]-[7].

\section{Conclusion}

The effects of antiretroviral drugs on the absorbance characteristics of HIV-infected blood have been studied. The methodology involved the serial dilution of the five different antiretroviral drugs (two HAART/FDC and three single drugs) and the subsequent incubation with the blood samples collected from ten HIV infected persons who have not yet commenced treatment with the antiretroviral drugs ten HIV infected persons who had already commenced treatment with the antiretroviral drugs and ten HIV negative persons, for the absorbance measurements using a digital Ultraviolet Visible MetaSpecAE1405031Pro Spectrophotometer. Reflectance, dielectric constant, etc. were derived from the absorbance data. For these drugs to be effective as HIV blockers, they should be able to coat the surfaces of the Lymphocytes. The question therefore arises as to what extent these drugs are able to coat the surfaces of the blood cells in HIV positive blood. This was established using the extent of absorbance change. Models for coating effectiveness were formulated and used to determine the coating effectiveness. Red blood cells were shown not to give reliable results. The results obtained however establish the fact that some coating of the drug had really occurred on the surfaces of the lymphocytes. Coating effectiveness was found to be most significant on the surfaces of uninfected lymphocytes, showing that HIV has the effect of reducing the surface area of blood cells. Blood of patients that had been on antiretroviral drug treatments was about $38 \%$ more effective in presence of drug 2 . The coating effectiveness of the lymphocytes was used to explain some observed clinical findings. Light absorption was shown to be most significant in HIV negative lymphocytes. HIV was seen therefore to have the effect of reducing the light absorption capacity of the blood cell. The use of the findings of this work in drug design may be expected to yield good results.

\section{References}

[1] Achebe, C.H. (2010) Human Immunodefficiency Virus (HIV)-Blood Interactions: Surface Thermodynamics Approach. Ph.D. Dissertation, Nnamdi Azikiwe University, Awka.

[2] United States Department of Health and Human Services (2004) A Guide to Primary Care for People with HIV/AIDS, 2004 Edition. Washington DC.

[3] Achebe, C.H. and Omenyi, S.N. (2013) The Effects of Human Immunodeficiency Virus (HIV) Infections on the Absorbance Characteristics of Different Blood Components. International Journal of Engineering and Science Invention, 2, 53-61. www.ijesi.org

[4] Ekwuagana, E., (2014) Oral Communication. Tahilah Diagnostic Laboratories, Awka, Anambra State, Nigeria.

[5] Ani, O.I. (2015) Surface Energetics Study of the Interactions between HIV and Blood Cells Treated with Antiretroviral Drugs. Ph.D. Dissertation, Nnamdi Azikiwe University, Awka.

[6] Ozoihu, E.M. (2014) Human Immunodefficiency Virus (HIV)-Blood Interactions: Contact Angle Approach. PhD. Dissertation, Nnamdi Azikiwe University, Awka.

[7] Achebe, C.H. and Omenyi, S.N. (2013) Mathematical Determination of the Critical Absolute Hamaker Constant of the Serum (as an Intervening Medium) Which Favours Repulsion in the Human Immunodeficiency Virus (HIV)-Blood Interactions Mechanism. Lecture Notes in Engineering and Computer Science. Proceedings of the World Congress on Engineering, WCE 2013, London, 3-5 July 2013, 1380-1384. 